\title{
Intrinsic Turbulence Stabilization in a Stellarator
}

\author{
P. Xanthopoulos, * G. G. Plunk, A. Zocco, and P. Helander \\ Max-Planck-Institut für Plasmaphysik, Wendelsteinstrasse 1, 17491 Greifswald, Germany
}

(Received 12 February 2016; published 7 June 2016)

\begin{abstract}
The magnetic surfaces of modern stellarators are characterized by complex, carefully optimized shaping and exhibit locally compressed regions of strong turbulence drive. Massively parallel computer simulations of plasma turbulence reveal, however, that stellarators also possess two intrinsic mechanisms to mitigate the effect of this drive. In the regime where the length scale of the turbulence is very small compared to the equilibrium scale set by the variation of the magnetic field, the strongest fluctuations form narrow bandlike structures on the magnetic surfaces. Thanks to this localization, the average transport through the surface is significantly smaller than that predicted at locations of peak turbulence. This feature results in a numerically observed upshift of the onset of turbulence on the surface towards higher ion temperature gradients as compared with the prediction from the most unstable regions. In a second regime lacking scale separation, the localization is lost and the fluctuations spread out on the magnetic surface. Nonetheless, stabilization persists through the suppression of the large eddies (relative to the equilibrium scale), leading to a reduced stiffness for the heat flux dependence on the ion temperature gradient. These fundamental differences with tokamak turbulence are exemplified for the QUASAR stellarator [G. H. Neilson et al., IEEE Trans. Plasma Sci. 42, 489 (2014)].
\end{abstract}

DOI: 10.1103/PhysRevX.6.021033

Over the last several decades, magnetic confinement fusion research has been dominated by two concepts, the tokamak and the stellarator. Most of the effort has gone into tokamaks, which employ axisymmetric magnetic fields to confine the plasma, but it has always been recognized that stellarators enjoy certain advantages, although they use more complicated fields and therefore face a number of technical challenges [1]. Compared to tokamaks, the physics of stellarator plasmas is less understood, and the greatest uncertainty concerns the nature of the plasma turbulence. This topic is of particular interest to the Wendelstein 7-X stellarator, which has recently been commissioned and completed its first-plasma milestone $[2,3]$. Its performance is expected to depend crucially on the turbulence present in the plasma, which will be explored thoroughly in upcoming experimental campaigns.

The great challenge for both tokamaks and stellarators is to provide stable and robust plasma confinement with minimal energy losses. The latter are caused by plasma turbulence and by so-called neoclassical transport, which results from the random walk taken by plasma particles moving along complicated orbits in the magnetic field while colliding with each other [4]. Neoclassical losses are modest in tokamaks but tend to be prohibitively large in

*pavlos.xanthopoulos@ipp.mpg.de

Published by the American Physical Society under the terms of the Creative Commons Attribution 3.0 License. Further distribution of this work must maintain attribution to the author(s) and the published article's title, journal citation, and DOI.
Subject Areas: Plasma Physics

stellarators, unless the magnetic field geometry is optimized to reduce the neoclassical transport [5], in which case the turbulent loss channel becomes relatively more important. Both for W7-X and other modern stellarators, such as QUASAR (based on the NCSX quasi-axisymmetric design by the Princeton Plasma Physics Laboratory, which to date has not been assembled) [6], the result of the optimization is a rather complex magnetic field [7]. The geometric properties of the field lines vary greatly over the magnetic surfaces, and the latter are pushed against each other in some locations, resulting in locally large temperature gradients and consequently strong drive for turbulence. The natural question that arises is how this feature affects the turbulence transport and how the latter compares to that in tokamaks.

Turbulence in tokamaks and stellarators is generally thought to be caused by low-frequency plasma instabilities, such as the electrostatic, collisionless ion temperature gradient (ITG) driven mode [8,9]. A theoretical method to understand and, ideally, predict the behavior of turbulence is furnished by "gyrokinetics" [10], according to which the fully kinetic description of the plasma is reduced by one dimension, owing to the fast gyration of the charged particles around the magnetic field lines. Despite this simplification, the coupled system of nonlinear partial differential equations is five dimensional (plus time) and can only be solved by numerical codes, such as GKV-X [11], GS2 [12], and GENE [13], with the help of modern supercomputers. We employ the massively parallel GENE code, which is able to address both tokamak (covering the entire plasma radius) [14] and stellarator (covering an entire 
magnetic surface, while radially local) [15] configurations. For all simulations shown, the plasma ions are treated gyrokinetically while the electrons are assumed to have a Boltzmann (adiabatic) distribution (the inclusion of gyrokinetic electrons is, at present, intractable because of the immense amount of computational resources required). The dimensionless parameter expressing the strength of the ITG turbulence drive is $1 / L_{T_{i}}=-1 / T_{i} d T_{i} / d s$ ( $T_{i}$ is the ion temperature). Here, $s=\sqrt{\Phi_{\text {tor }} / \Phi_{\text {lcfs }}} \in[0,1]$ is the radial coordinate ( $\Phi_{\text {tor }}$ is the toroidal magnetic flux, with $\Phi_{\text {lcfs }}$ its value at the outermost surface); for all simulations, we have selected $s=0.7$, which corresponds to a radius where turbulence is expected to be particularly active. Although several physical mechanisms for ITG turbulence stabilization have been reported in tokamak-related gyrokinetic simulations, for instance, involving electromagnetic effects $[16,17]$ or geometric effects like triangularity [18], here we address intrinsic stabilization effects that are due solely to the nonaxisymmetric magnetic geometry. Specifically, the pivotal point in the present work is that, in a stellarator, the transport averaged over a magnetic surface can be significantly lower than the transport produced locally at the most unstable regions on the surface.

The effects we consider encompass a wide range of values for the dimensionless parameter $\rho^{*}=\rho / a$, where $\rho$ is the ion gyroradius and $a$ the minor radius of the torus. In the "small- $\rho^{*}$ regime" characterized here by $\rho^{*}=1 / 250$, the turbulence scale is much smaller than the scale set by the variation of the equilibrium magnetic field. Turbulence thus appears localized on the surface, in that the strongest fluctuations accumulate along thin bandlike structures, as shown in Fig. 1 for the QUASAR configuration. This

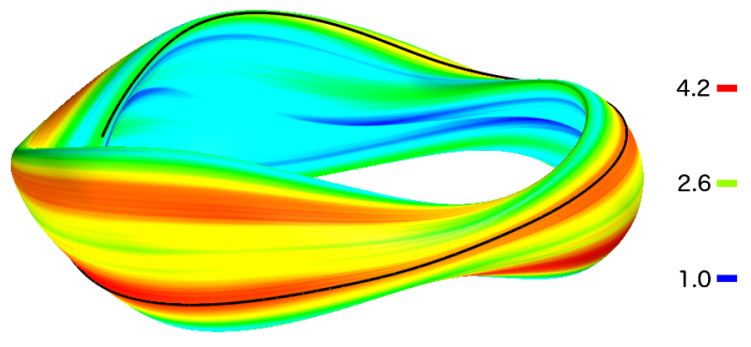

FIG. 1. Root-mean-squared normalized density fluctuations caused by ITG turbulence $\left(1 / L_{T_{i}}=2\right)$ on the magnetic surface of the QUASAR stellarator in the small- $\rho^{*}$ regime. The strongest fluctuations appear localized around the magnetic field line with $\alpha=\pi / 3$ (black), and similarly for the line with $\alpha=-\pi / 3$ (not shown), because of the symmetry of the configuration. The number of grid points in the five-dimensional phase space $\left(x, y, z, v_{\|}, \mu\right)$ is $128 \times 128 \times 128 \times 64 \times 10$. The simulation box size for the radial direction is $L_{x}=147 \rho$ and for the binormal direction $L_{y}=204 \rho$. Also, $L_{z}=2 \pi$ for the parallel direction (parametrized by the poloidal angle). The simulation box size for the parallel velocity is $-3 v_{t} \leq v_{\|} \leq 3 v_{t}$ and for the magnetic moment $0 \leq \mu \leq 9 T_{i} / B_{0}$. Here, $v_{t}=\sqrt{2 T_{i} / m_{i}}$ is the thermal velocity and $B_{0}=2 \Phi_{\mathrm{lcfs}} / a^{2}$.

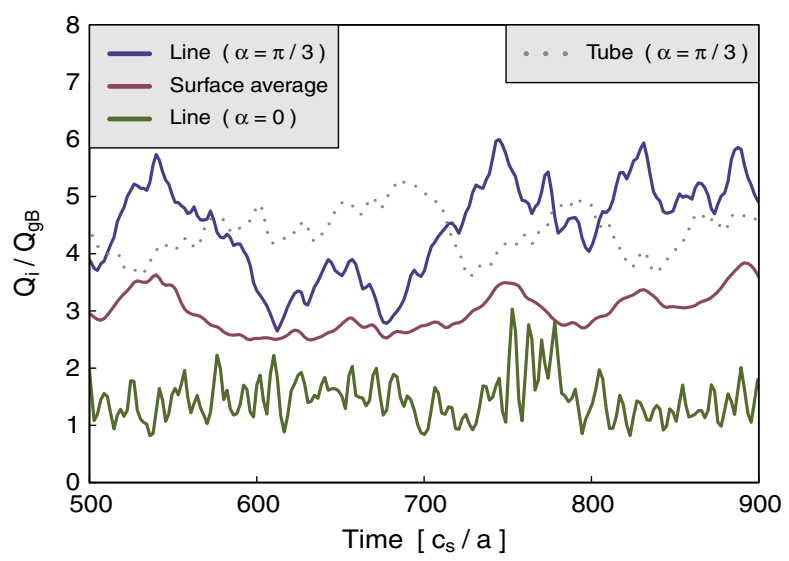

FIG. 2. Ion heat flux (in gyroBohm units) caused by ITG turbulence $\left(1 / L_{T_{i}}=2\right)$ on the magnetic surface of the QUASAR stellarator in the small- $\rho^{*}$ regime. The time traces correspond to the field line with $\alpha=\pi / 3$ (strong turbulence region), the field line with $\alpha=0$ (weak turbulence region), and the surface average, demonstrating the stabilization (averaging) effect. Also shown is the time trace from a separate flux tube simulation surrounding the field line with $\alpha=\pi / 3$, suggesting the local nature of stellarator turbulence in small- $\rho^{*}$ conditions.

localization is responsible for a reduction of the transport through the surface as compared with its local maximum since large relatively quiescent regions bring down the average level. Figure 2 presents ion heat flux time traces in gyroBohm units, $Q_{\mathrm{gB}}=c_{s} P\left(\rho^{*}\right)^{2}\left(c_{s}=\sqrt{T_{i} / m_{i}}\right.$ is the ion sound speed and $P$ is the plasma pressure). The time traces for the most unstable magnetic field line with $\alpha=\pi / 3$ and the surface average are displayed. The coordinate $\alpha=$ $\zeta-q \theta$ labels a magnetic field line covering one poloidal turn; $\theta, \zeta$ are the poloidal and toroidal angles, and $q$ is the safety factor, defining the amount of winding of the magnetic field line on the surface. Also shown in Fig. 2 is the time trace from a location of weak turbulence, at the magnetic line with $\alpha=0$. It is found that the resulting transport on the surface lies between the two field line levels, demonstrating the averaging effect. There is an important observation concerning the small $-\rho^{*}$ regime, namely that, despite the deviation of the surface-averaged transport from the strongest local one, ITG turbulence still behaves locally with respect to a field line. Indeed, if we use a slender computational domain surrounding a single field line, a so-called "flux tube" [19], then locality is to be understood in the sense that the transport stemming from a magnetic field line on the surface is equal to that produced separately, using a flux tube around this line. We stress, however, that locality in the stellarator does not imply that the transport calculated from a single flux tube can predict the surface-averaged transport.

To appreciate the critical role of nonaxisymmetry for turbulence stabilization, we can compare Fig. 1 with Fig. 3, corresponding to a tokamak with a similar aspect ratio (3.87) as QUASAR (4.47). Evidently, in the small- $\rho^{*}$ 


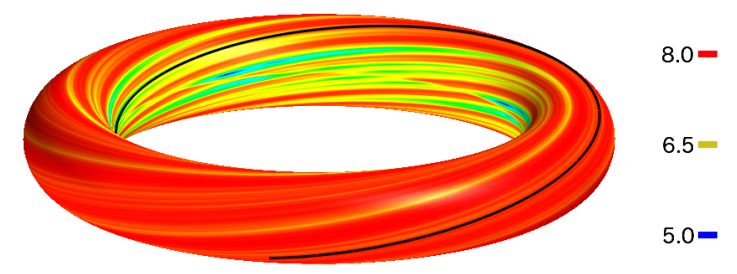

FIG. 3. Root-mean-squared normalized density fluctuations caused by ITG turbulence $\left(1 / L_{T_{i}}=2\right)$ on the magnetic surface of a tokamak in the small- $\rho^{*}$ regime. For these parameters, $\rho^{*}=4 / 250$ is found to be adequate to obtain the small- $\rho^{*}$ limit. Unlike the stellarator in the same regime, strong fluctuations cover the entire outboard side of the surface, where the excitation of the ITG turbulence is favored.

regime, no localization of the ITG turbulence exists in the tokamak since the axisymmetric geometry induces an almost even distribution of the fluctuations on the outboard side of the surface. As a result, the surface-averaged heat flux is essentially the same as the heat flux measured at various field lines, implying a lack of this stabilization for the tokamak (see Fig. 4). The beneficial turbulence localization in QUASAR could be lost, however, in the "large- $\rho$ * regime" characterized here by $\rho^{*}=1 / 125$, where the ITG density fluctuations occupy the entire outboard domain of the configuration (see Fig. 5). In this regime, the distribution of turbulent intensity resembles that of a tokamak. Be that as it may, ITG turbulence still enjoys stabilization, as another mechanism applies, once $\rho^{*}$ increases, via interaction of turbulent eddies with the rapidly varying magnetic field in a stellarator. A theoretical explanation of this mechanism can be obtained in the fluid limit, by examining the linear drive of the electrostatic fluctuations $\phi(\alpha)$ of the ITG mode with frequency $\omega$,

$$
\frac{T_{i}}{T_{e}} \omega^{2} \phi=\omega_{d} \omega_{*}^{\mathrm{T}} \frac{\partial^{2} \phi}{\partial \alpha^{2}},
$$

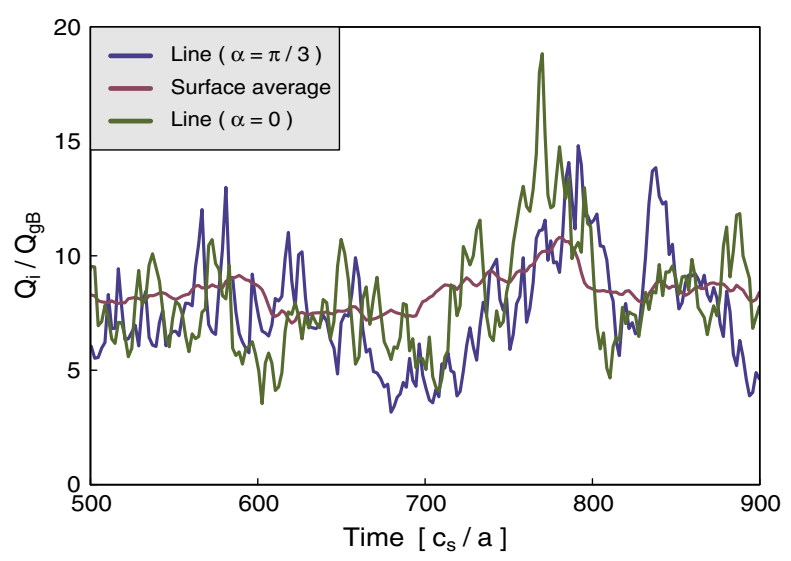

FIG. 4. Ion heat flux (in gyroBohm units) caused by ITG turbulence $\left(1 / L_{T_{i}}=2\right)$ on the magnetic surface of a tokamak in the small- $\rho^{*}$ regime. The time traces correspond to two different magnetic field lines and the surface average, all exhibiting almost the same transport level.

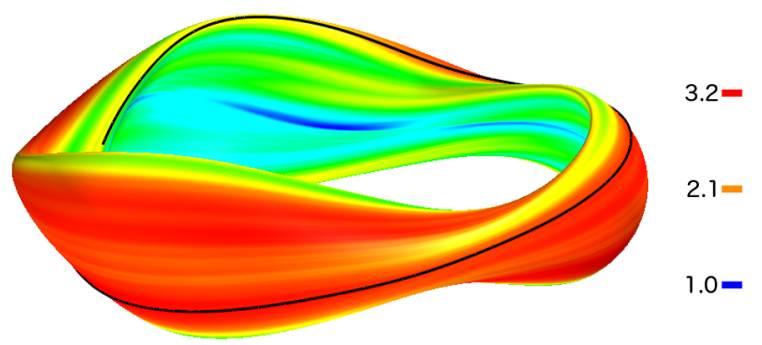

FIG. 5. Root-mean-squared normalized density fluctuations caused by ITG turbulence $\left(1 / L_{T_{i}}=2\right)$ on the magnetic surface of the QUASAR stellarator in the large- $\rho^{*}$ regime. The localization pattern is lost and the fluctuations appear spread out on the surface, as in the tokamak case.

where $T_{e}$ is the electron temperature, $e$ is the charge, $\omega_{*}^{\mathrm{T}}=\left(q T_{i} / e\right) d \ln T_{i} / d \Phi_{\text {tor }}$, and the magnetic drift frequency reading $\omega_{d}=\boldsymbol{\nabla} \alpha \cdot(\mathbf{b} \times \boldsymbol{\nabla} \ln B) 2 T_{i} /(e B)$ introduces nonaxisymmetry via its field line dependence (b is the magnetic field unit vector and $B$ the magnetic field intensity). In a stellarator, geometric quantities such as magnetic shear and curvature cause the magnetic drift to vary in $\alpha$ (unlike in a tokamak), so it exhibits areas both favoring $\left(\omega_{d}<0\right)$ and suppressing $\left(\omega_{d}>0\right)$ local instability. To model this effect, we can consider an analytically tractable form of $\omega_{d}(\alpha)$ given by a periodic, piecewise constant function, alternating between regions of positive and negative values, with the extent of these regions representing the equilibrium scale associated with the variation of $\omega_{d}$. The solutions of Eq. (1) are in this case waves $\left(\partial_{\alpha} \rightarrow i k_{\alpha}\right)$, but since $\omega_{d}$ multiplies the derivative term, finiteness of $\partial^{2} \phi / \partial^{2} \alpha$ requires that zeros in the ITG eigenmode $\phi(\alpha)$ occur where zeros of $\omega_{d}$ exist. Thus, in analogy to a standing wave on a string, a minimum allowable wave number $k_{\alpha}$ is imposed, here determined by the equilibrium scale of the magnetic drift. In short, the size of the mode is limited by the size of the unstable region where it resides. At small $\rho^{*}$ this is of no consequence since the length scale of the turbulence is much smaller than the equilibrium scale. At sufficiently large $\rho^{*}$ though, this scale separation is lost, and the lower bound on $k_{\alpha}$ implies that large eddies, which would carry most of the fluctuation energy, are suppressed. In a tokamak, turbulence stabilization due to $\rho^{*}$ is expected to be much weaker compared to the stellarator (radial effects that can additionally influence the tokamak transport [20] are not taken into account): Although axisymmetric configurations formally also impose a geometric constraint on the scale of turbulence, this is merely set by toroidal periodicity, rather than smaller-scale 3D magnetic shaping. As a consequence, significant turbulence stabilization for the tokamak requires much larger gradients and $\rho^{*}$ values compared to the stellarator. This is evidenced by Fig. 6, which serves as a summary of the effect of the turbulence stabilization mechanisms in QUASAR and the tokamak. For the stellarator, ion heat flux scaling curves are shown as a 


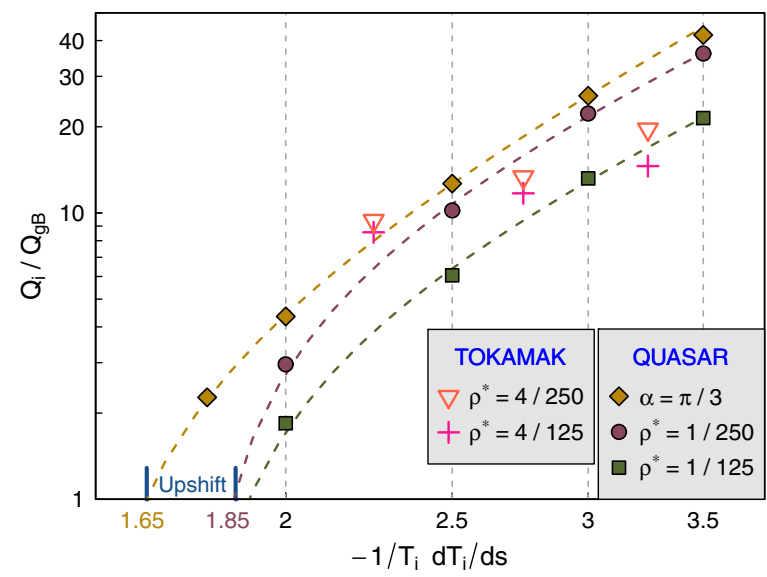

FIG. 6. Scaling of the ion heat flux (in gyroBohm units) caused by ITG turbulence on the magnetic surface of the QUASAR stellarator and the tokamak in the two $\rho^{*}$ regimes. Also shown is the curve corresponding to the local turbulence at the most unstable field line with $\alpha=\pi / 3$ on the magnetic surface of QUASAR. The established upshift (about 15\%) is due to the turbulence averaging on the surface.

function of the ion temperature gradient, for the two values of $\rho^{*}$, together with the curve corresponding to the most unstable magnetic field line $(\alpha=\pi / 3)$ on the surface. We note that stabilization in the large- $\rho^{*}$ regime primarily causes a favorable reduction of the transport stiffness (the rate at which the transport increases), while in the small- $\rho^{*}$ regime, stabilization is mainly responsible for the upshift observed by comparing the threshold of the surfaceaveraged transport to that of the most active field line. We emphasize that this effect is solely related to the nonaxisymmetric geometry, and note, as Fig. 4 suggests, that no such upshift should be reported in tokamak gyrokinetic simulations as the heat flux levels for the surface and any magnetic field line are practically identical (to avoid clutter, these data points do not appear in Fig. 6).

The newly observed upshift is comparable in magnitude but otherwise unrelated to the well-known Dimits shift $[21,22]$ in the context of ITG turbulence, which refers to the difference between the linear and nonlinear thresholds; the upshift in Fig. 6, instead, involves two nonlinear scalings. In fact, a typical Dimits shift is also found in QUASAR, by comparing the local linear stability threshold $(\approx 1.50$ in Fig. 7) with the nonlinear threshold $(\approx 1.65$ in Fig. 6) measured on the field line. As this nonlinear scaling is extracted from the surface simulation, we may conclude that zonal flow activity should also be present on the magnetic surface. Interestingly, however, the linear threshold of the surface mode $(\approx 1.85$ in Fig. 7) appears to coincide with the small- $\rho^{*}$ nonlinear threshold of the surface transport. This apparent contradiction is resolved by noting that the global linear mode stabilization should not be related to nonlinear stabilization (heat flux reduction) as the global linear mode involves communication across the entire flux surface, contrary to the local behavior

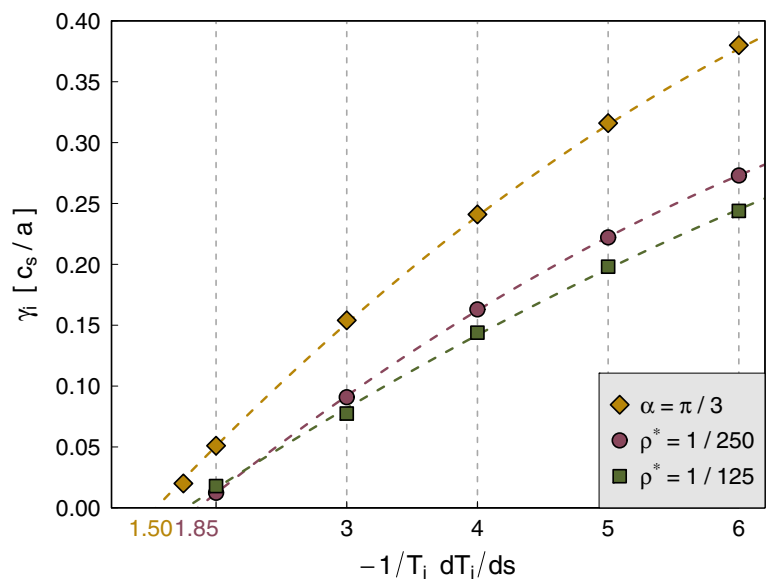

FIG. 7. Scaling of the linear growth rate for the ITG mode on the magnetic surface of the QUASAR stellarator (in the two $\rho^{*}$ regimes) and the field line with $\alpha=\pi / 3$.

of turbulence in the small- $\rho^{*}$ regime. In this context, we also warn against inferring a direct connection between the upshift of the nonlinear threshold and the corresponding linear one. As demonstrated in Fig. 8, even in the large- $\rho^{*}$ regime, the scale of the corresponding linear mode is very different from the turbulence scale. For that matter, one can observe that the heat flux reduction is disproportionately large relative to the growth rate reduction.

Summarizing, we have highlighted two different mechanisms by which a stellarator is able to stabilize ITG-driven turbulence on the surface, solely thanks to nonaxisymmetry. The first mechanism applies in the small- $\rho^{*}$ regime, where ITG fluctuations are localized in the vicinity of certain magnetic field lines on the surface, whereas the second is relevant in the large- $\rho^{*}$ regime, where the fluctuations are distributed more evenly distributed on a large portion of the surface. The first stabilization mechanism is due to the averaging between regions of strong and weaker turbulence

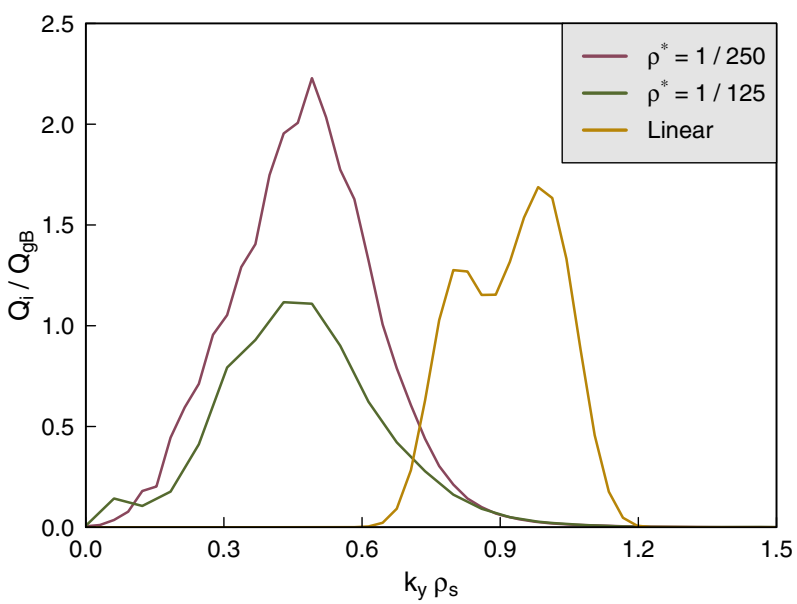

FIG. 8. Heat flux spectra for QUASAR in the two $\rho^{*}$ regimes $\left(1 / L_{T_{i}}=3\right)$ compared to a linear fluctuation spectrum (rescaled; $\rho^{*}=1 / 250$ ), showing the difference between dominant scales. 
and, relative to the threshold of the most turbulent field line, additionally involves a newly observed upshift. The transport difference stemming from the surface and the most unstable location might serve as an additional figure of merit for the characterization of turbulence optimization in a stellarator [23]. In addition, because of the very small electron gyroradius, it is highly likely that turbulence caused by the electron temperature gradient will also experience stabilization in the small- $\rho^{*}$ regime. The other type of stabilization occurs because of the suppression of large energy-containing eddies by the fast variation of the equilibrium magnetic field, and it leads to a reduction of the transport stiffness. Because underlying mechanisms are quite generic, both stabilization mechanisms are expected to also act in other types of stellarators, where the "poloidal" localization of turbulence is either theoretically predicted [15] or experimentally verified [24]. Finally, we note that $3 \mathrm{D}$ geometrical turbulence stabilization could also be introduced in a tokamak if magnetic perturbations break the axisymmetry, thereby enhancing the local shear [25] and confining turbulence structures, similar to the stellarator.

The GENE simulations were performed on the Helios (Japan) and Hydra (Germany) supercomputers. We thank J. Geiger and Y. Turkin for technical support with the magnetic equilibria.

[1] P. Helander, C. D. Beidler, T. M. Bird, M. Drevlak, Y. Feng, R. Hatzky, F. Jenko, R. Kleiber, J. H. E. Proll, Y. Turkin, and P. Xanthopoulos, Stellarator and Tokamak Plasmas: A Comparison, Plasma Phys. Controlled Fusion 54, 124009 (2012).

[2] T. Klinger et al., Towards Assembly Completion and Preparation of Experimental Campaigns of Wendelstein 7-X in the Perspective of a Path to a Stellarator Fusion Power Plant, Fusion Eng. Des. 88, 461 (2013).

[3] T. S. Pedersen et al., Plans for the First Plasma Operation of Wendelstein 7-X, Nucl. Fusion 55, 126001 (2015).

[4] P. Helander and D. J. Sigmar, Collisional Transport in Magnetized Plasmas (Cambridge University Press, Cambridge, England, 2002).

[5] C. D. Beidler, K. Allmaier, M. Yu. Isaev, S. V. Kasilov, W. Kernbichler, G. O. Leitold, H. Maaßberg, D. R. Mikkelsen, S. Murakami, M. Schmidt, D. A. Spong, V. Tribaldos, and A. Wakasa, Benchmarking of the Mono-energetic Transport Coefficients. Results from the International Collaboration on Neoclassical Transport in Stellarators (ICNTS), Nucl. Fusion 51, 076001 (2011).

[6] G. H. Neilson, D. A. Gates, P. J. Heitzenroeder, J. Breslau, S. C. Prager, T. Stevenson, P. Titus, M. D. Williams, and M. C. Zarnstorff, Next Steps in Quasi-Axisymmetric Stellarator Research, IEEE Trans. Plasma Sci. 42, 489 (2014).

[7] A. Boozer and S. Design, J. Plasma Phys. 81, 515810606 (2015).

[8] W. Horton Jr., D. Choi, and W. M. Tang, Toroidal Drift Modes Driven by Ion Pressure Gradients, Phys. Fluids 24, 1077 (1981).
[9] G. G. Plunk, P. Helander, P. Xanthopoulos, and J. W. Connor, Collisionless Microinstabilities in Stellarators III. The Ion-Temperature-Gradient Mode, Phys. Plasmas 21, 032112 (2014).

[10] A. J. Brizard and T. S. Hahm, Foundations of Nonlinear Gyrokinetic Theory, Rev. Mod. Phys. 79, 421 (2007).

[11] M. Nunami, T. Watanabe, and H. Sugama, Gyrokinetic Vlasov Code Including Full Three-Dimensional Geometry of Experiments, Plasma Fusion Res. 5, 016 (2010).

[12] http://sourceforge.net/projects/gyrokinetics.

[13] F. Jenko, W. Dorland, M. Kotschenreuther, and B. N. Rogers, Electron Temperature Gradient Driven Turbulence, Phys. Plasmas 7, 1904 (2000); http://genecode.org.

[14] T. Görler, X. Lapillonne, S. Brunner, J. Chowdhury, T. Dannert, F. Jenko, B. F. McMillan, F. Merz, D. Told, and L. Villard, Nonlocal Effects in Gyrokinetic Turbulence Simulations using GENE, J. Phys. 260, 1 (2010).

[15] P. Xanthopoulos, H. E. Mynick, P. Helander, Y. Turkin, G. G. Plunk, F. Jenko, T. Görler, D. Told, T. Bird, and J. H. E. Proll, Controlling Turbulence in Present and Future Stellarators, Phys. Rev. Lett. 113, 155001 (2014).

[16] H. Doerk, M. Dunne, F. Jenko, F. Ryter, P. A. Schneider, E. Wolfrum, and The ASDEX Upgrade Team, Electromagnetic Effects on Turbulent Transport in High-Performance ASDEX Upgrade Discharges, Phys. Plasmas 22, 042503 (2015).

[17] J. Citrin, F. Jenko, P. Mantica, D. Told, C. Bourdelle, J. Garcia, J. W. Haverkort, G. M. D. Hogeweij, T. Johnson, and M. J. Pueschel, Nonlinear Stabilization of Tokamak Microturbulence by Fast Ions, Phys. Rev. Lett. 111, 155001 (2013).

[18] Y. Camenen, A. Pochelon, R. Behn, A. Bottino, A. Bortolon, S. Coda, A. Karpushov, O. Sauter, G. Zhuang, and the TCV Team, Impact of Plasma Triangularity and Collisionality on Electron Heat Transport in TCV L-Mode Plasmas, Nucl. Fusion 47, 510 (2007).

[19] M. A. Beer, S. C. Cowley, and G. W. Hammett, FieldAligned Coordinates for Nonlinear Simulations of Tokamak Turbulence, Phys. Plasmas 2, 2687 (1995).

[20] B. F. McMillan, X. Lapillonne, S. Brunner, L. Villard, S. Jolliet, A. Bottino, T. Görler, and F. Jenko, System Size Effects on Gyrokinetic Turbulence, Phys. Rev. Lett. 105, 155001 (2010).

[21] A. M. Dimits et al., Comparisons and Physics Basis of Tokamak Transport Models and Turbulence Simulations, Phys. Plasmas 7, 969 (2000).

[22] D. R. Mikkelsen and W. Dorland, Dimits Shift in Realistic Gyrokinetic Plasma-Turbulence Simulations, Phys. Rev. Lett. 101, 135003 (2008).

[23] H. Mynick, P. Xanthopoulos, B. Faber, M. Lucia, M. Rorvig, and J. N. Talmadge, Turbulent Optimization of Toroidal Configurations, Plasma Phys. Controlled Fusion 56, 094001 (2014).

[24] G. Birkenmeier, M. Ramisch, P. Manz, B. Nold, and U. Stroth, Experimental Investigation of the Magnetic Configuration Dependence of Turbulent Transport, Phys. Rev. Lett. 107, 025001 (2011).

[25] C. C. Hegna, The Effect of Three-Dimensional Fields on Bounce Averaged Particle Drifts in a Tokamak, Phys. Plasmas 22, 072510 (2015). 